\title{
Phase I Study of Tandem High-Dose Chemotherapy With Autologous Peripheral Blood Stem Cell Rescue for Children With Recurrent Brain Tumors: A Pediatric Blood and Marrow Transplant Consortium Study
}

\author{
Andrew L. Gilman, MD, ${ }^{1 *}$ Chad Jacobsen, $M D,{ }^{1}$ Nancy Bunin, $\mathrm{MD}^{2}$ John Levine, $\mathrm{MD},{ }^{3}$ Fred Goldman, $\mathrm{MD},{ }^{4}$ \\ Anne Bendel, $\mathrm{MD}^{5}$ Michael Joyce, $\mathrm{MD}^{6}{ }^{6}$ Peter Anderson, MD, PhD, ${ }^{7}$ Marta Rozans, $\mathrm{MD}, \mathrm{PhD}^{8}$ Donna A. Wall, $\mathrm{MD},{ }^{9}$ \\ Tobey J. MacDonald, $\mathrm{MD},{ }^{10}$ Steve Simon, $\mathrm{PhD},{ }^{11}$ and Richard P. Kadota, $\mathrm{MD}^{12}$
}

Background. High-dose chemotherapy with autologous stem cell rescue (HDC/SCR) has produced responses and prolonged survival for some children with recurrent brain tumors, but is associated with considerable morbidity and mortality. A Phase I trial of two cycles of HDC/SCR for recurrent brain tumors in children was performed to determine the maximum tolerated doses for a novel regimen. Procedures. Two cycles of HDC/SCR were given. Cycle 1 included thiotepa and carmustine given on days $-5,-4$, and -3 . Four to six weeks later, patients received cycle 2 which included thiotepa and carboplatin given on days $-5,-4$, and -3 . Autologous peripheral blood stem cells (PBSC) were infused on day 0 of each cycle. Results. Thirty-two patients weretreated and 25 patients received both cycles of HDC/SCR. Common toxicities included mucositis, emesis, diarrhea, anorexia, and pancytopenia. Eight of 32 (25\%) assessable children died from regimen-related toxicity. Pulmonary failure occurred in seven patients. Seven patients had grade 3-4 neurotoxicity. The 3-year eventfree survival (EFS) was $25 \%$. Conclusions. We determined the maximum tolerated regimen to be thiotepa $600 \mathrm{mg} / \mathrm{m}^{2}$ and carmustine $300 \mathrm{mg} / \mathrm{m}^{2}$ followed by thiotepa $600 \mathrm{mg} / \mathrm{m}^{2}$ and carboplatin $1,200 \mathrm{mg} / \mathrm{m}^{2}$. Pulmonary toxicity was considerable. The toxic death rate was similar to other trials of HDC/SCR for children with recurrent brain tumors performed during the same time period. The regimen resulted in prolonged time to progression for a significant number of patients and long-term survival for some patients with recurrent medulloblastoma and rhabdoid tumor. Pediatr Blood Cancer 2011;57:506513. (C) 2010 Wiley-Liss, Inc.

Key words: brain tumors; myeloablative therapy; Phase I clinical trials; stem cell transplantation

\section{INTRODUCTION}

Brain tumors are the most common solid tumor of childhood. The outcome of children with recurrent malignant brain tumors is dismal [1]. High-dose chemotherapy followed by autologous peripheral blood stem cell rescue (HDC/SCR) has been used for the treatment of recurrent brain tumors. Several chemotherapy combinations have been studied. While toxicities have been significant, this approach has resulted in long-term survival with good quality of life for some patients [2-7]. Patients with gross residual disease at the time of HDC/SCR and those with metastatic disease are much more likely to have disease progression despite this approach [8-13]. Radiation therapy prior to HDC/SCR may also be associated with a worse outcome [13-17]. There have also been several studies of 2-4 cycles of HDC/SCR reported for children with high-risk newly diagnosed brain tumors [18-20] or recurrent disease [6,21-23].

We report the results of a Phase I trial of tandem HDC/SCR for recurrent brain tumors in children. The goal was to improve outcomes with less toxicity by using two cycles of HDC/SCR at reduced intensity compared to a single cycle of HDC/SCR. The novel chemotherapy regimen used alkylating agents - thiotepa, carboplatin, and carmustine — which have activity against brain tumors [3,10,24]. Dose escalation of thiotepa in both cycles and of carmustine was planned. Table I compares the drug doses used for this study versus those reported for a single HDC/SCR approach by the Children's Cancer Group (CCG) [10,24].

\section{METHODS}

\section{Patient Eligibility}

Patients were eligible if they had a progressive or recurrent brain tumor including medulloblastoma (MB), ependymoma, high-grade (III/IV) astrocytoma (HGA), primitive neuroectodermal tumor (PNET), rhabdoid tumor, or pineoblastoma. Patients with brain stem tumors and those who had previous HDC/SCR were not eligible. Other eligibility criteria included: (1) age 1-21 years, (2) availability of at least $4 \times 10^{6} \mathrm{CD} 34+$ cells $/ \mathrm{kg}$ of body weight available for SCR, (3) creatinine clearance $>50 \mathrm{ml} / \mathrm{min} / 1.73 \mathrm{~m}^{2}$ or serum creatinine $<2 \times$ upper limit of normal, (4) bilirubin $<1.5 \mathrm{mg} / \mathrm{dl}$ and SGPT $<5 \times$ upper limit of normal, (5) $\mathrm{FEV}_{1}, \mathrm{FVC}$, and DLCO (corrected) $\geq 60 \%$ of predicted (no dyspnea on exertion and oxygen saturation $\geq 95 \%$ by pulse oximetry on room air if unable to perform spirometry), (6) cardiac ejection fraction of $\geq 50 \%$ (by echo or MUGA), (7) absolute neutrophil count $>1,000 / \mu 1$ and platelet count $>100,000 / \mu 1$, (8) performance score (Lansky or Karnofsky) of $\geq 50$, (9) no treatment (radiation or chemotherapy) within 3 weeks of protocol therapy, excluding steroid therapy for increased intracranial pressure. The study was approved by the Internal Review Committee of the Pediatric Blood and Marrow Transplant Consortium as well as by the Institutional Review Board of participating institutions and informed consent was obtained from the patient or guardian.

${ }^{1}$ Levine Children's Hospital, Charlotte, North Carolina; ${ }^{2}$ Children's Hospital of Philadelphia, Philadelphia, Pennsylvania; ${ }^{3}$ University of Michigan, Ann Arbor, Michigan; ${ }^{4}$ Children's Hospital of Alabama, Birmingham, Alabama; ${ }^{5}$ Children's Hospitals and Clinics of Minnesota, Minneapolis/St. Paul, Minnesota; ${ }^{6}$ Nemour's Children's Clinic, Jacksonville, Florida; ${ }^{7}$ University of Texas MD Anderson Cancer Center, Houston, Texas; ${ }^{8}$ St. Christopher's Hospital for Children, Philadelphia, Pennsylvania; ${ }^{9}$ CancerCare Manitoba, Winnipeg, Manitoba, Canada; ${ }^{10}$ Children's Healthcare of Atlanta/ Emory University, Atlanta, Georgia; ${ }^{11}$ Children's Mercy Hospital, Kansas City, Missouri; ${ }^{12}$ Rady Children's Hospital, San Diego, California

\section{Conflict of interest: Nothing to declare.}

*Correspondence to: Andrew L. Gilman, MD, Levine Children's Hospital, Carolinas Medical Center, 1000 Blythe Blvd., Charlotte, NC 28203.

E-mail: andrew.gilman@ carolinashealthcare.org

Received 24 August 2010; Accepted 8 October 2010 
TABLE I. Comparison of Drug Doses Between This Study and Prior Children's Cancer Group (CCG) HDC/SCR Studies

\begin{tabular}{lcccc}
\hline & & & \multicolumn{2}{c}{ Current regimen $\left(\mathrm{mg} / \mathrm{m}^{2}\right)$} \\
\cline { 5 - 5 } & $\left(\mathrm{mg} / \mathrm{m}^{2}\right)$ & $\left(\mathrm{mg} / \mathrm{m}^{2}\right)$ & Cycle 1 & Cycle 2 \\
\hline Thiotepa & 900 & 900 & 600 & $600-750$ \\
Etoposide & 750 & 750 & & \\
BCNU & & 600 & 300 & \\
Carboplatin & 1,500 & & & $1,200-1,500$ \\
\hline
\end{tabular}

\section{Patient Characteristics}

Patient characteristics are presented in Table II. Thirty-three patients were enrolled on the study. One patient developed progressive disease prior to study therapy and no further information is reported. The median patient age was 7 years (range 1.75-18). The median time from diagnosis to progressive disease prior to HDC was 21 months (range 3-120). The median time from progressive disease to HDC was 3 months (range 1-10).

Eighteen patients had MB, 15 with a history of metastatic disease within the CNS, including 10 with spinal drop metastases. Chang staging for 17 patients for whom data are available were M0 (3), M2 (5), M3 (10). Other tumors included HGA (7), ependymoma (3), rhabdoid tumor (2), supratentorial PNET (1), and pineoblastoma (1). Twenty-five patients had residual disease at the time of HDC/SCR. Twelve patients had bulky tumor (defined as $>1 \mathrm{~cm}$ in greatest diameter) at the time of HDC/SCR, including seven patients with MB.

\section{Study Design}

The study was a Phase I trial conducted by the Pediatric Blood and Marrow Transplant Consortium. Patients were enrolled from 1995 to 2002. Patients had collection of PBSC prior to study entry. PBSC containing $\geq 2 \times 10^{6} \mathrm{CD} 34+$ cells $/ \mathrm{kg}$ were infused on day 0 of each cycle. The first HDC cycle included thiotepa IV over $3 \mathrm{hr}$ followed immediately by carmustine IV over $2 \mathrm{hr}$ on days $-5,-4$, and -3 . Dexamethasone $4 \mathrm{mg} / \mathrm{m}^{2}$ daily PO or IV was given on days $-5,-4$, and -3 in an attempt to prevent pulmonary toxicity from BCNU. The second HDC cycle included thiotepa IV over $3 \mathrm{hr}$ followed immediately by carboplatin IVover 2 hron days $-5,-4$, and -3 . For patients with a creatinine clearance of $<100 \mathrm{ml} / \mathrm{min} / 1.73 \mathrm{~m}^{2}$, the dose of carboplatin was calculated using a modified Calvert formula [25,26]. The target AUC used was 7 and $5.5 \mathrm{mg} \mathrm{min} / \mathrm{ml}$ for the corresponding doses of 500 and $400 \mathrm{mg} / \mathrm{m}^{2}$, respectively. The lower of the dose calculated by per $\mathrm{m}^{2}$ dosing or by the modified Calvert formula was used.

Cycle 2 commenced between 28 and 42 days after the PBSC infusion of cycle 1 if: (1) ANC was $\geq 500 / \mu l$ and platelets were $\geq 20,000 / \mu 1$ (could be with transfusion support); (2) non-hematopoietic toxicity has resolved sufficiently; (3) study eligibility criteria for renal, hepatic, and pulmonary function were met at this time. Local radiotherapy was allowed after the second PBSC cycle when the platelet count reached $50,000 / \mu 1$.

\section{Dose Escalation and Maximum Tolerable Regimen (MTR)}

Dose escalation was planned as shown in Table III. Patients were treated in cohorts of three. Toxicity was graded according to the
Children's Cancer Group Toxicity scale. If none of three patients had dose-limiting toxicity (DLT), subsequent patients were enrolled at the next dose level. If one out of three had DLT, then an additional three patients were enrolled at the same dose level. If two patients at a dose level had DLT, then that level was deemed unacceptable and an additional three patients were treated at the previous level. If $<2$ out of 6 patients had DLT, the dose level was considered tolerable. DLT was defined as: (1) severe organ dysfunction according to Bearman criteria [27] lasting $>7$ days, or; (2) death attributable to regimenrelated toxicity occurring before day 28 following SCR (day 100 for non-infectious pulmonary toxicity). Patients were considered not assessable for determination of the MTR and were replaced if they received only one HDC/SCR and did not have DLT.

When the protocol was amended to allow the enrollment of an additional six patients at the MTR in order to better define the pulmonary toxicity at this level and to modify the steroid dosing during the conditioning regimen, the following rule was added. For the six additional patients, if two experience DLT, the study will be closed and the previous MTR without the minor modification will be deemed the acceptable approach.

\section{Clinical Monitoring and Intervention for Lung Disease}

Because of pulmonary toxicity associated with high-dose carmustine, patients were monitored with handheld spirometry daily and pulse oximetry weekly starting with cycle 1 and continuing for 4 weeks following cycle 2 . A decrease in spirometry volume by $>10 \%$ or oxygen saturation (by $>3 \%$ and/or to $<95 \%$ ) was followed by pulmonary function testing if the patient could comply. Significant decreases in lung volumes or diffusion capacity were treated with prednisone 1-2 mg/kg/day, which could be tapered over 2 months (as tolerated) following improvement.

\section{Statistical Analysis}

Overall and event-free survival was calculated from the day of SCR after the first HDC cycle. The survivor function was estimated by the method of Kaplan and Meier [28]. Survival curves were created using the program R (R Development Core Team, Vienna, Austria).

\section{RESULTS}

\section{Therapy}

Seven patients received only one cycle of HDC/SCR because of severe toxicity (1), toxic death (3), disease progression (1), and parental request without severe toxicity (2). Two patients received RT for residual disease after HDC/SCR (one craniospinal and one local RT) as allowed by the protocol. Two patients received temozolomide after recovery from HDC/SCR because of bulky residual disease.

\section{Toxicity}

Common toxicities included mucositis, emesis, diarrhea, anorexia, and pancytopenia. Mucositis was usually mild to moderate; 5/ 32 patients had grade 3-4 mucositis. Mucositis was usually more severe after the second cycle. Emesis and diarrhea were usually mild to moderate; $2 / 32$ patients had grade $3-4$ diarrhea and 1 patient had grade 4 emesis. Anorexia frequently required parenteral nutrition. Twelve bacterial infections occurred in nine patients, including 
Gilman et al.

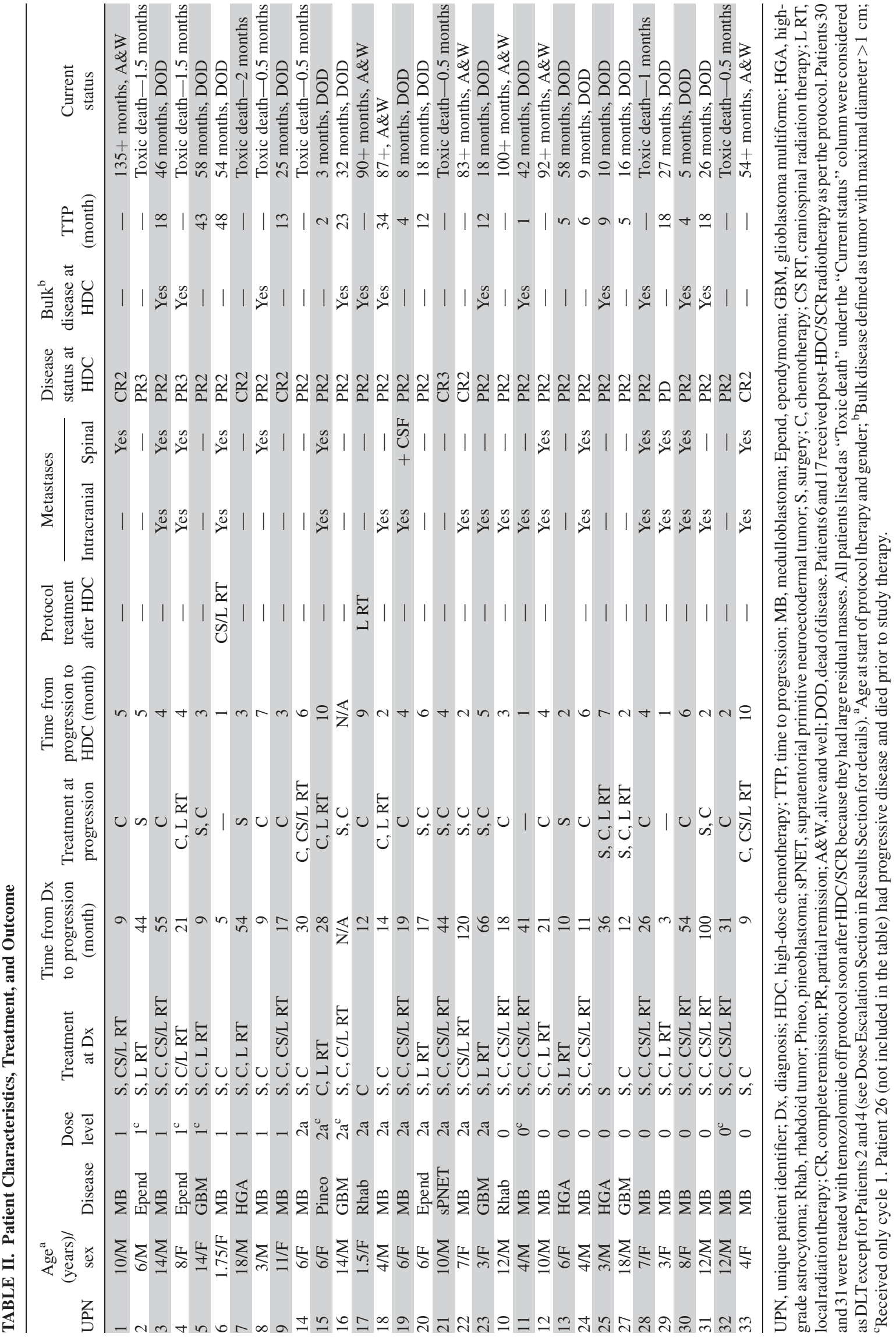


TABLE III. Dose Escalation Schema

\begin{tabular}{|c|c|c|}
\hline Dose level & First cycle & Second cycle \\
\hline Level 0 & $\begin{array}{l}\text { Thiotepa } 600 \mathrm{mg} / \mathrm{m}^{2} \\
\text { BCNU } 300 \mathrm{mg} / \mathrm{m}^{2}\end{array}$ & $\begin{array}{l}\text { Thiotepa } 600 \mathrm{mg} / \mathrm{m}^{2} \\
\text { Carboplatin } 1.200 \mathrm{mg} / \mathrm{m}^{2}\end{array}$ \\
\hline Level 1 & $\begin{array}{l}\text { Thiotepa } 600 \mathrm{mg} / \mathrm{m}^{2} \\
\text { BCNU } 300 \mathrm{mg} / \mathrm{m}^{2}\end{array}$ & $\begin{array}{l}\text { Thiotepa } 600 \mathrm{mg} / \mathrm{m}^{2} \\
\text { Carboplatin } \mathbf{1 , 5 0 0} \mathbf{~ m g} / \mathbf{m}^{2}\end{array}$ \\
\hline Level 2a & $\begin{array}{l}\text { Thiotepa } 600 \mathrm{mg} / \mathrm{m}^{2} \\
\text { BCNU } 300 \mathrm{mg} / \mathrm{m}^{2}\end{array}$ & $\begin{array}{l}\text { Thiotepa } 750 \mathbf{~ m g} / \mathbf{m}^{2} \\
\text { Carboplatin } 1,200 \mathrm{mg} / \mathrm{m}^{2}\end{array}$ \\
\hline Level 3a & $\begin{array}{l}\text { Thiotepa } 750 \mathrm{mg} / \mathrm{m}^{2} \\
\text { BCNU } 300 \mathrm{mg} / \mathrm{m}^{2}\end{array}$ & $\begin{array}{l}\text { Thiotepa } 750 \mathrm{mg} / \mathrm{m}^{2} \\
\text { Carboplatin } 1,200 \mathrm{mg} / \mathrm{m}^{2}\end{array}$ \\
\hline Level 4a & $\begin{array}{l}\text { Thiotepa } 750 \mathrm{mg} / \mathrm{m}^{2} \\
\text { BCNU } 450 \mathbf{~ m g} / \mathbf{m}^{2}\end{array}$ & $\begin{array}{l}\text { Thiotepa } 750 \mathrm{mg} / \mathrm{m}^{2} \\
\text { Carboplatin } 1,200 \mathrm{mg} / \mathrm{m}^{2}\end{array}$ \\
\hline
\end{tabular}

The dose escalated at each level is shown in bold. The original dose of carboplatin for levels $2-4$ was $1,500 \mathrm{mg} / \mathrm{m}^{2}$. However, because of doselimiting toxicity at level 1 (see text), the dose of carboplatin was decreased when therapy was de-escalated to dose level 0 . The dose of carboplatin remained at $1,200 \mathrm{mg} / \mathrm{m}^{2}$ for the amended dose levels $2-4$.

bacteremia ( 8 gram positive and 3 gram negative) and one catheterrelated Pseudomonas cellulitis.

Grade 3-5 toxicities and grade 2 pulmonary toxicity treated with steroids are shown in Table IV. No patient had non-fatal severe organ dysfunction lasting $>7$ days. Eight patients (25\%) had regimenrelated deaths. The cause of death was pulmonary failure $(n=4$, including two with pulmonary hypertension), pulmonary and renal failure $(n=2)$, renal failure $(n=1)$, and severe hepatic veno-occlusive disease (VOD) with multi-organ failure (MOF) $(\mathrm{n}=1)$. Fatal pulmonary toxicity was more common at dose level 1, occurring in 4/ 9 patients at this level compared to $2 / 23$ patients at the other dose levels. Non-fatal pulmonary toxicity occurred in eight patients. Four patients had restrictive lung disease without an oxygen requirement and four patients required oxygen. One episode was associated with cytomegalovirus reactivation and one episode that occurred 5 months after HDC/SCR was associated with Pneumocystis jirovecii after craniospinal RT. The non-infectious cases resolved with steroid therapy. Three patients who required steroids for pulmonary toxicity after cycle 1 , tolerated cycle 2 and did well long-term. Seven subjects had grade 3-4 neurotoxicity, one with concomitant VOD. Severe neurological toxicity included coma (2), seizures (3), ataxia (1), and weakness/lethargy (1). Three patients with pre-existing sensorineural hearing loss (SNHL) had worsening SNHL after HDC/SCR.

\section{Dose Escalation}

Two of six assessable patients at dose level 1 had DLT. Both had MOF, one with VOD. Three additional patients treated at dose level 1 were considered not assessable for the purpose of determining the MTR. One patient received only the first HDC/SCR cycle per parental request. Two patients had very complicated histories and clinical courses that confounded the role of HDC in their deaths. One patient died due to aspiration pneumonia and with refractory seizures presumed to be due to a familial seizure disorder that had also claimed the life of his sister. The second patient had pulmonary failure associated with aspiration 3 weeks following cycle 1 after esophageal dilatation for a chronic stricture.

The dose level was de-escalated to level 0 by reducing the carboplatin dose in cycle 2 . As the fatal pulmonary toxicities seen at dose level 1 occurred soon after course 2 chemotherapy, we attributed them to the intensity of cycle 2 therapy and not to the nitrosourea. We therefore kept the nitrosourea dose the same in dose level 0 as in dose level 1. After 3/3 assessable patients tolerated dose level 0 , the dose level was then escalated to dose level 2a (modification of the original dose level 2 using the reduced carboplatin dose of level 0 with an increase in the thiotepa dose during cycle 2). Only one of three assessable patients treated at dose level $2 \mathrm{a}$ had DLT, so an additional three patients were enrolled. Because of previous experience with some patients not being assessable, two additional patients were enrolled in anticipation of the need for replacement patients. When two of the first six had DLT (one VOD with MOF and one pulmonary failure), dose level $2 \mathrm{a}$ was deemed unacceptable. The additional two patients did not have DLT.

Dose level 0 was further evaluated with the enrollment of an additional three patients according to the study design. None of the first six patients at dose level 0 had DLT. Because $2 / 6$ patients had nonfatal pulmonary toxicity requiring steroid therapy, an additional six patients were enrolled at dose level 0 to better define the risk of pulmonary toxicity. In addition, steroid therapy during carmustine administration was increased from dexamethasone $4 \mathrm{mg} / \mathrm{m}^{2}$ daily on day -5 to day -3 to $4 \mathrm{mg} / \mathrm{m}^{2}$ twice daily on day -5 to day -1 for these patients in an attempt to prevent pulmonary toxicity. Despite this change, two of these six children had DLT (one MOF and one pulmonary failure). Both patients with DLT had Pseudomonas infections; one episode was between cycles 1 and 2 and the other followed cycle 2 . It is possible that the additional steroid therapy contributed to

TABLE IV. Severe Toxicities by Dose Level

\begin{tabular}{|c|c|c|c|c|c|c|c|c|c|}
\hline $\begin{array}{l}\text { Dose } \\
\text { level }\end{array}$ & Pulmonary & Hepatic & Renal & Neurological & MOF & Infection & Mucositis & GI & Skin \\
\hline 0 & $\begin{array}{c}5 / 13 \\
(1 \mathrm{Gr} 2,3 \text { Gr } 3 \\
1 \text { Gr } 5)\end{array}$ & $0 / 13$ & $\begin{array}{c}1 / 13 \\
(1 \text { Gr } 5)\end{array}$ & $\begin{array}{c}3 / 13 \\
(2 \mathrm{Gr} 3,1 \mathrm{Gr} 4)\end{array}$ & $0 / 13$ & $\begin{array}{c}7 / 13 \\
(5 \mathrm{Gr} 3, \\
2 \mathrm{Gr} 4)\end{array}$ & $\begin{array}{c}3 / 13 \\
(2 \mathrm{Gr} 3, \\
1 \mathrm{Gr} 4)\end{array}$ & $\begin{array}{c}2 / 13 \\
(1 \mathrm{Gr} 3, \\
1 \mathrm{Gr} 4)\end{array}$ & $0 / 13$ \\
\hline 1 & $\begin{array}{c}5 / 9 \\
(1 \mathrm{Gr} 3,4 \text { Gr 5) }\end{array}$ & $\begin{array}{c}1 / 9 \\
(1 \mathrm{Gr} 3)\end{array}$ & $\begin{array}{c}1 / 9 \\
(1 \mathrm{Gr} 4)\end{array}$ & $\begin{array}{c}2 / 9 \\
(1 \mathrm{Gr} 3,1 \mathrm{Gr} 4)\end{array}$ & $2 / 9$ & $\begin{array}{c}2 / 9 \\
(2 \mathrm{Gr} 3)\end{array}$ & $\begin{array}{c}2 / 9 \\
(2 \mathrm{Gr} 3)\end{array}$ & $0 / 9$ & $\begin{array}{c}1 / 9 \\
(1 \mathrm{Gr} 4)\end{array}$ \\
\hline $2 \mathrm{a}$ & $\begin{array}{c}4 / 10 \\
(1 \mathrm{Gr} 2,1 \mathrm{Gr} 3, \\
1 \mathrm{Gr} 4,1 \mathrm{Gr} 5)\end{array}$ & $\begin{array}{c}1 / 10 \\
(1 \text { Gr } 5)\end{array}$ & $\begin{array}{c}1 / 10 \\
(1 \mathrm{Gr} 4)\end{array}$ & $\begin{array}{c}2 / 10 \\
(1 \mathrm{Gr} 3,1 \mathrm{Gr} 4)\end{array}$ & $2 / 10$ & $\begin{array}{c}1 / 10 \\
(1 \mathrm{Gr} 3)\end{array}$ & $0 / 10$ & $\begin{array}{c}1 / 10 \\
(1 \mathrm{Gr} 4)\end{array}$ & $\begin{array}{c}1 / 10 \\
(1 \mathrm{Gr} 3)\end{array}$ \\
\hline
\end{tabular}

MOF, multi-organ failure; GI, gastrointestinal. Severe toxicities include grade 3-5 toxicities and grade 2 pulmonary toxicities requiring steroid therapy; grade 5 toxicity is fatal. Multi-organ failure includes patients in whom the primary cause of death was hepatic (i.e., veno-occlusive disease) or pulmonary failure. Two of the four fatal pulmonary toxicities at dose level 1 were judged to not be related to study therapy (see text). 
the toxic deaths by causing increased immunosuppression. Overall, $2 / 12$ assessable patients treated at dose level 0 had DLT and the toxic death rate was $17 \%$.

\section{Patient Outcome}

Thirty-two patients received protocol therapy. Although the study was a Phase I study to determine an acceptable treatment regimen, long-term outcome data are available. Seven children remain alive and without disease, including 5/18 with $\mathrm{MB}(135,92,87,83$, and 54 months after HDC/SCR) and both patients with rhabdoid tumors (100 and 90 months after HDC/SCR). All five surviving patients with MB had metastatic disease prior to HDC/SCR, including three with spinal leptomeningeal disease. All of them had received RT prior to HDC/SCR - craniospinal (two at diagnosis and one at disease progression) or local (one at diagnosis and one at disease progression). Only one of them had bulk disease at the time of HDC. This patient is alive and well 87 months after HDC, despite progressive disease 34 months after HDC. The patient had resection of a large drop metastasis that confirmed relapsed MB. Following surgery, the patient was treated with chemotherapy and local radiotherapy. Eighteen patients had progressive disease at a median of 12 months after HDC/SCR (range 1-48).

Progression-free and overall survival curves are shown in Figure 1. The Kaplan-Meier estimates of EFS and overall survival at 3 years for all patients are $25 \%$ (95\% CI 14-46\%) and 38\% (95\% CI $24-59 \%)$. The Kaplan-Meier estimates of EFS and overall survival at 3 years for patients with MB are $28 \%$ (95\% CI 13-58\%) and $44 \%$ (95\% CI 26-74\%).

\section{DISCUSSION}

HDC/SCR has been shown to be beneficial for some patients with recurrent brain tumors, with 3-year EFS of $\sim 30 \%$ in some reports [2]. We conducted a Phase I trial of tandem cycles of HDC. The intensity of each myeloablative cycle was reduced to avoid excessive cumulative organ toxicity. The first cycle was intended to be cytoreductive, allowing the second cycle to be delivered at a time of minimal residual disease. Another unique feature of the regimen is that alkylating agents were given on the same days rather than on sequential days as in previous trials of HDC/SCR. The hypothesis was that efficacy would be increased by achieving higher peak alkylator levels and toxicity would be decreased (especially mucositis) due to the shorter duration of exposure. A dose-escalation study design was used to determine doses with acceptable acute and cumulative toxicities.

The use of two cycles of thiotepa allowed the total thiotepa dose to be escalated from $900 \mathrm{mg} / \mathrm{m}^{2}$ as previously reported with HDC/SCR for brain tumors [8] to $1,200 \mathrm{mg} / \mathrm{m}^{2}$. However, carboplatin dosing had to be reduced compared to that previously reported [10]. The incidence of pulmonary toxicity was higher than expected for carmustine at the dose given [29]. The latter two findings may have been related to giving the alkylating agents on the same day.

The tandem HDC/SCR therapy was associated with considerable toxicity. Pulmonary toxicity was a major limitation of this therapy, occurring in $44 \%$ of patients. It should be noted that our assessment for pulmonary toxicity is intentionally very inclusive and includes patients with aspiration pneumonia, infections, and post-infectious complications that likely were not directly due to the specific chemotherapy used. A similar $45 \%$ incidence of pulmonary toxicity was reported for a myeloablative regimen containing carmustine $600 \mathrm{mg} /$ $\mathrm{m}^{2}$, thiotepa, and etoposide for children with newly diagnosed HGA [24]. In our study, pulmonary toxicity occurred at all dose levels, but was most severe at dose level 1 . There was only 1 fatal pulmonary toxicity out of 13 patients at the MTR (dose level0) and this was due to pulmonary hypertension following Pseudomonas sepsis. An association between either gender or prior craniospinal irradiation and nitrosourea-related pulmonary toxicity has been previously described. We found no statistical association between pulmonary toxicity and either gender $(P=1)$ or prior spinal irradiation $(P=0.5)$.
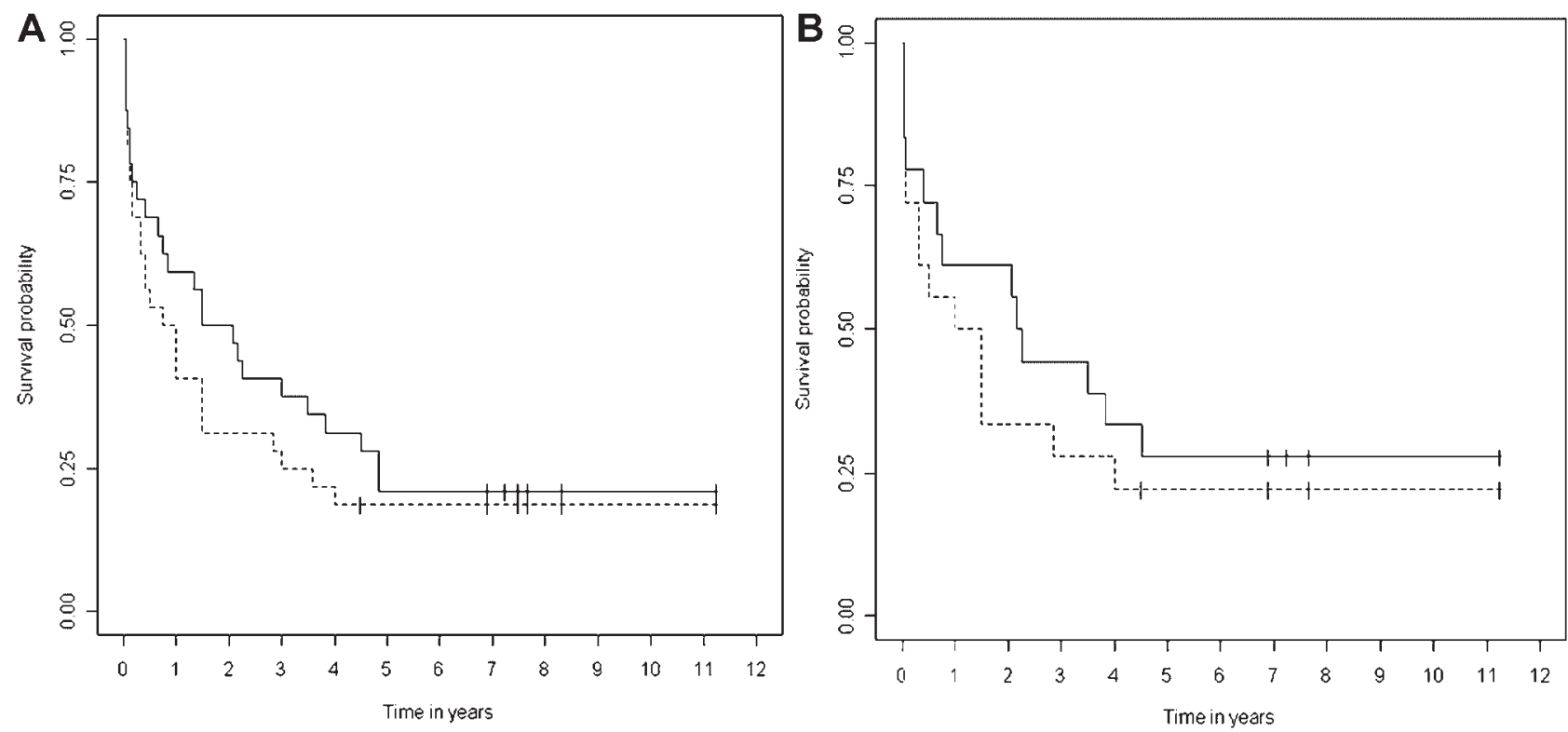

Fig. 1. A: Event-free survival (- - -) and overall survival ( with medulloblastoma. 
Neurotoxicity occurred in $22 \%$ of patients and was similar at all dose levels. Neurotoxicity has been reported at similar frequencies with other regimens for brain tumors-30\% with busulfan and thiotepa [30] and $22 \%$ with etoposide, thiotepa, and carboplatin [10]. Grade 3-4 hepatotoxicity occurred in $6 \%$ of patients, which compares favorably to $25 \%$ reported with busulfan and thiotepa [9] and $56 \%$ reported with etoposide, thiotepa, and carboplatin [10]. Severe renal toxicity occurred in $6 \%$ patients, similar to that reported with etoposide, thiotepa, and carboplatin [10]. Mucositis and gastrointestinal toxicity were less common than reported with other regimens for recurrent brain tumors [10,11]. This finding supports our hypothesis that fewer days of chemotherapy administration would reduce mucosal toxicity.

Three patients had worsening SNHL after HDC/SCR. All had medullobastoma, had received craniospinal irradiation with posterior fossa boost, and had SNHL prior to HDC/SCR. The relationship to prior therapy has been reported with other high-dose carboplatin regimens [21].

Our tandem HDC/SCR regimen resulted in similar toxicities to a single cycle of etoposide, thiotepa, and carboplatin [3,10]. Possible advantages of the tandem regimen include less severe mucositis and less grade 3-4 hepatic toxicity. Unfortunately, the toxic death rate of $25 \%$ in our Phase I trial was similar to Phase II trials using one cycle of HDC which reported rates of 11-22\% [10,12,18,31]. It should be noted that the toxic death rate in our study was $17 \%$ at the MTR and $0 \%$ for the six patients treated at the MTR without the additional steroid therapy (as discussed above). More recent studies have demonstrated toxic mortality rates declining to below $10 \%$ [32]. We would point out that our study was conducted between 1995 and 2002. As such we would attribute the discrepancy between the toxic death rates in contemporary studies and in ours to ongoing improvements in supportive care for such heavily pretreated patients. Also, the toxic death rate in our Phase I study and the toxic death rates from Phase II studies are difficult to compare because there are likely significant differences in the disease status and prior therapy between patients treated on these different types of studies.

A preliminary assessment of efficacy is possible although it must be noted that this is in the context of a Phase I trial that was not designed to look at efficacy. The 3-year actuarial event-free survival (EFS) for patients with MB was $28 \%$ (5/18). All five surviving patients with MB had metastatic disease prior to HDC/SCR, including three with spinal leptomeningeal disease. All of them had received RT prior to $\mathrm{HDC} / \mathrm{SCR}$ - craniospinal (two at diagnosis and one at disease progression) or local (one at diagnosis and one at disease progression). Of course, the RT at disease progression could have contributed to the long-term disease control. Efficacy of our approach is also suggested by the prolonged progression-free interval experienced by a significant number of patients. In addition to the long-term survivors, $36 \%$ of patients had progression-free intervals of at least 1 year after SCR including 5/13 patients with $\operatorname{MB}(13,18,18,18,48$ months), 3/6 with glioblastoma multiforme or HGA (12, 23, and 43 months), and $1 / 3$ with ependymoma (12 months). Of note, only two of these patients received additional therapy during the progression-free interval. One patient received craniospinal RT with a local boost for residual disease as allowed per protocol. The other patient received temozolomide for residual bulky disease.

The discussion of efficacy is very complicated based on numerous factors. This study was one of the few Phase I dose escalation trials performed for HDC/SCR in children. Most studies have used fixed doses of chemotherapy for pilot or Phase II studies. Because our study was a Phase I trial of a novel chemotherapy regimen, we had an extremely high-risk cohort of patients. For example, 15/18 MB patients had metastatic disease and 7/18 had bulk disease at the time of transplant. Also, of the five long-term survivors with MB, three did not have biopsy of recurrent disease or neurological symptoms at the time of recurrence. These three patients had recurrence at 9, 14, and 21 months after diagnosis. It is possible that some of the radiographic relapses represented imaging changes rather than disease. Of note, Dunkel et al. [15] did not find a statistically significant difference in EFS for patients with MRI evidence of recurrent MB versus those with tissue confirmation of recurrence. Interestingly, our results are remarkably similar to those of Dunkel for patients with recurrent MB $[10,15]$.

Thirteen of 18 patients with MB had received radiotherapy as part of their initial treatment. Three of $13(23 \%)$ previously irradiated patients and 2/5 (40\%) non-irradiated patients survive. It should be noted that some studies have demonstrated similar survival rates in previously irradiated patients $[13,15]$, whereas others have reported dismal results in such patients $[16,17,33]$.

Tandem HDC/SCR did not overcome the impact of bulk disease. At dose level 0, the maximum tolerated regimen, disease-free survival is $3 / 5$ patients without bulk disease compared to $0 / 4$ with bulk disease at the time of HDC. Bulk disease has been correlated with poor outcome in most HDC/SCR studies [32].

Metastatic disease at HDC/SCR for patients with $\mathrm{MB}$ is another poor prognostic factor. In a study of etoposide, thiotepa, and carboplatin for recurrent MB, the 3-year EFS was $26 \%$ for patients with $\mathrm{M}^{+}$disease and $60 \%$ for patients with M0 disease [10]. Similarly, a report of melphalan and cyclophosphamide for recurrent MB had 4/4 patients surviving after HDC/SCR for patients with relapse isolated to the posterior fossa, but $0 / 14$ survivors with $\mathrm{M} 2^{+}$disease [11]. In our study, 5/15(33\%) patients with $\mathrm{M} 2^{+}$disease remain alive with follow-up of 54-135 months.

The two long-term survivors with rhabdoid tumors are noteworthy in light of the very poor prognosis for such patients. One patient had intracranial metastases at the time of progression and had received craniospinal RT at initial diagnosis. The other patient had bulky disease at the primary site at the time of HDC. This patient received only local RT after HDC/SCR because of young age. Finally, despite prolonged time to progression in three patients with glioblastoma multiforme, the long-term survival for patients with HGA and other histologies was poor. This is similar to the experience for these tumors reported with other HDC regimens, although there is some suggestion of benefit in some reports.

In conclusion, we determined the maximum tolerated regimen for tandem HDC/SCR with carmustine and thiotepa for the first cycle and thiotepa and carboplatin for the second cycle. Although mucositis and hepatotoxicity were less common and less severe compared to reports of single cycle HDC/SCR, pulmonary toxicity was considerable and the toxic death rate was not improved compared to that of a single cycle of HDC/SCR in patients with recurrent disease performed during the same time period. If a Phase II study with chemotherapy at the MTR is performed in the future, it will be important to closely monitor pulmonary toxicity and to have appropriate stopping rules for toxicity. The tandem regimen appears to have significant activity against brain tumors, particularly medulloblastomas and rhabdoid tumors. 


\section{ACKNOWLEDGMENT}

The authors would like to thank Dr. Ira Dunkel for his critical review of the manuscript. Patients were enrolled at the following PBMTC institutions: Alberta Children's Hospital, Calgary, Alberta; Children's Hospitals and Clinics of Minnesota, Minneapolis/St. Paul, MN; Children's Hospital of Philadelphia, Philadelphia, PA; Children's Hospital Medical Center, Cincinnati, OH; Children's Mercy Hospital, Kansas City, MO; Children's National Medical Center, Washington, DC; DeVos Children's Hospital, Grand Rapids, MI; Mayo Clinic, Rochester, MN; Methodist Children's Hospital of South Texas, San Antonio, TX; Nemour's Children's Clinic, Jacksonville, FL; Tulane University, New Orleans, LA; University of Iowa, Iowa City, IA; University of Michigan, Ann Arbor, MI.

\section{REFERENCES}

1. Gillheeny SW, Khakoo Y, Souweidane M, et al. Thiotepa/topotecan/ carboplatin with autoplogous stem cell rescue in recurrent/refractory/poor prognosis pediatric malignancies of the central nervous system. Pediatr Blood Cancer 2010;54:591-595.

2. Dunkel IJ, Finlay JL. High-dose chemotherapy with autologous stem cell rescue for brain tumors. Crit Rev Oncol Hematol 2002;41:197-204.

3. Gururangan S, Dunkel IJ, Goldman S, et al. Myeloablative chemotherapy with autologous bone marrow rescue in young children with recurrent brain tumors. J Clin Oncol 1998;16:2486-2493.

4. Grodman H, Wolfe L, Kretschmar C. Outcome of patients with recurrent medulloblastoma or central nervous system germinoma treated with low dose continuous intravenous etoposide along with dose-intensive chemotherapy followed by autologous hematopoietic stem cell rescue. Pediatr Blood Cancer 2009;53:33-36.

5. Kadota RP, Mahoney DH, Doyle J, et al. Dose intensive melphalan and cyclophosphamide with autologous hematopoietic stem cells for recurrent medulloblastoma or germinoma. Pediatr Blood Cancer 2008;51:675-678.

6. Sung KW, Yoo KH, Cho EJ, et al. High-dose chemotherapy and autologous stem cell rescue in children with newly diagnosed highrisk or relapsed medulloblastoma or supratentorial primitive neuroectodermal tumor. Pediatr Blood Cancer 2007;48:408-415.

7. Finlay JL, Dhall G, Boyett JM, et al. Myeloablative chemotherapy with autologous bone marrow rescue in children and adolescents with recurrent malignant astrocytoma: Outcome compared with conventional chemotherapy: A report from the Children's Oncology Group. Pediatr Blood Cancer 2008;51:806-811.

8. Finlay JL, Goldman S, Wong MC, et al. Pilot study of high-dose thiotepa and etoposide with autologous bone marrow rescue in children and young adults with recurrent CNS tumors. J Clin Oncol 1996;14:2495-2503.

9. Dupuis-Girod S, Hartmann O, Benhamou E, et al. Will high dose chemotherapy followed by autologous bone marrow transplantation supplant cranio-spinal irradiation in young children treated for medulloblastoma. J Neurooncol 1996;27:87-98.

10. Dunkel IJ, Boyett JM, Yates A, et al. High-dose carboplatin, thiotepa, and etoposide with autologous stem-cell rescue for patients with recurrent medulloblastoma. J Clin Oncol 1998;16:222228.

11. Graham ML, Herndon JE, Casey JR, et al. High-dose chemotherapy with autologous stem-cell rescue in patients with recurrent and high-risk pediatric brain tumors. J Clin Oncol 1997;15:18141823.

12. Broniscer A, Nicolaides TP, Dunkel IJ, et al. High-dose chemotherapy with autologous stem-cell rescue in the treatment of patients with non-cerebellar primitive neuroectodermal tumors. Pediatr Blood Cancer 2004;42:261-267.

13. Butturini AM, Jacob M, Aguajo J, et al. High-dose chemotherapy and autologous hematopoietic progenitor cell rescue in children with recurrent medulloblastoma and supratentorial primitive neuroectodermal tumors-The impact of prior radiotherapy on outcome. Cancer 2009;115:2956-2963.

14. Gajjar A, Pizer B. Role of high-dose chemotherapy for recurrent medulloblastoma and other CNS primitive neuroectodermal tumors. Pediatr Blood Cancer 2010;54:649-651.

15. Dunkel IJ, Gardner SL, Garvin JH, et al. High-dose carboplatin, thiotepa, and etoposide with autologous stem cell rescue for patients with previously irradiated recurrent medulloblastoma. NeuroOncology 2010;12:297-303.

16. Gururangan S, Krauser J, Watral MA, et al. Efficacy of high-dose chemotherapy or standard salvage therapy in patients with recurrent medulloblastoma. Neuro-Oncology 2008;10:745-751.

17. Valteau-Couanet D, Fillipini B, Benhamou E, et al. High-dose busulfan and thiotepa followed by autologous stem cell transplantation (ASCT) in previously-irradiated medulloblastoma patients: High toxicity and lack of efficacy. Bone Marrow Transplant 2005;36:939-945.

18. Strother D, Ashley D, Kellie SJ, et al. Feasibility of four consecutive high-dose chemotherapy cycles with stem-cell rescue for patients with newly diagnosed medulloblastoma or supratentorial primitive neuroectodermal tumor after craniospinal radiotherapy: Results of a collaborative study. J Clin Oncol 2001;19:2696-2704.

19. Gajjar A, Chintagumpala M, Ashley D, et al. Risk-adapted craniospinal radiotherapy followed by high-dose chemotherapy and stemcell rescue in children with newly diagnosed medulloblastoma ( $\mathrm{St}$ Jude Medulloblastoma-96): Long term results from a prospective, multicentre trial. Lancet Oncol 2006;7:813-820.

20. Thorarinsdottir HK, Rood B, Kamani N, et al. Outcome for children $<4$ years of age with malignant central nervous system tumors treated with high-dose chemotherapy and autologous stem cell rescue. Pediatr Blood Cancer 2007;48:278-284.

21. Foreman NK, Schissel D, Le T, et al. A study of sequential high dose cyclophosphamide and high dose carboplatin with peripheral stemcell rescue in resistant or recurrent pediatric brain tumors. J Neurooncol 2005;71:181-187.

22. Ozkaynak MF, Sandoval C, Levendoglu-Tugal O, et al. A pilot trial of tandem autologous peripheral blood progenitor cell transplantation following high-dose thiotepa and carboplatin in children with poor-risk central nervous system tumors. Pediatr Hematol Oncol 2002;21:635-645.

23. Rosenfeld A, Kletzel M, Duerst R, et al. A phase II prospective study of sequential myeloablative chemotherapy with hematopoietic stem cell rescue for the treatment of selected high risk and recurrent central nervous system tumors. J Neurooncol 2010; 97:247-255.

24. Grovas AC, Boyett JM, Lindsley K, et al. Regimen-related toxicity of myeloablative chemotherapy with BCNU, thiotepa, and etoposide followed by autologous stem cell rescue for children with newly-diagnosed glioblastoma multiforme: Report from the Children's Cancer Group. Med Pediatr Oncol 1999;33:83-87.

25. Calvert AH, Newell DR, Gumbrell LA, et al. Carboplatin dosage: Prospective validation of a simple formula based on renal function. J Clin Oncol 1989;7:1748-1756.

26. Newell DR, Pearson ADJ, Balmanno K, et al. Carboplatin pharmacokinetics in children: The development of a pediatric dosing formula. J Clin Oncol 1993;11:2314-2323.

27. Bearman SI, Appelbaum FR, Buckner CD, et al. Regimen-related toxicity in patients undergoing bone marrow transplantation. J Clin Oncol 1988;6:1562-1568.

28. Kaplan EL, Meier P. Nonparametric estimation from incomplete observations. J Am Stat Assoc 1958;53:451-481. 
29. Ager S, Mahendra P, Richards EM, et al. High-dose carmustine, etoposide, and melphalan ('BEM') with autologous stem cell transplantation: A dose-toxicity study. Bone Marrow Transplant 1996; 17:335-340.

30. Kalifa C, Hartmann O, Vassal G, et al. High-dose busulfan and thiotepa with autologous bone marrow transplantation in childhood malignant brain tumors: A phase II study. Bone Marrow Transplant 1992;9:227-233.

31. Mahoney DH, Jr., Strother D, Camitta B, et al. High-dose melphalan and cyclophosphamide with autologous bone marrow rescue for recurrent/progressive malignant brain tumors in children: A pilot Pediatric Oncology Group study. J Clin Oncol 1996;14:382-388.

32. Marachelian A, Butturini A, Finlay J. Myeloablative chemotherapy with autologous hematopoietic progenitor cell rescue for childhood central nervous system tumors. Bone Marrow Transplant 2008;41: 167-172.

33. Massimino M, Gandola L, Spreafico F, et al. No salvage using highdose chemotherapy plus/minus reirradiation for relapsing previously irradiated medulloblastoma. Int J Radiat Oncol Biol Phys 2009;73:1358-1363. 